\title{
Prioritizing disease candidate genes by a gene interconnectedness-based approach
}

\author{
Chia-Lang Hsu', Yen-Hua Huang ${ }^{2}$, Chien-Ting Hsu' ${ }^{1}$, Ueng-Cheng Yang ${ }^{1,3^{*}}$ \\ From Asia Pacific Bioinformatics Network (APBioNet) Tenth International Conference on Bioinformatics - First \\ ISCB Asia Joint Conference 2011 (InCoB/ISCB-Asia 2011) \\ Kuala Lumpur, Malaysia. 30 November - 2 December 2011
}

\begin{abstract}
Background: Genome-wide disease-gene finding approaches may sometimes provide us with a long list of candidate genes. Since using pure experimental approaches to verify all candidates could be expensive, a number of network-based methods have been developed to prioritize candidates. Such tools usually have a set of parameters pre-trained using available network data. This means that re-training network-based tools may be required when existing biological networks are updated or when networks from different sources are to be tried.

Results: We developed a parameter-free method, interconnectedness (ICN), to rank candidate genes by assessing the closeness of them to known disease genes in a network. ICN was tested using 1,993 known disease-gene associations and achieved a success rate of $\sim 44 \%$ using a protein-protein interaction network under a test scenario of simulated linkage analysis. This performance is comparable with those of other well-known methods and ICN outperforms other methods when a candidate disease gene is not directly linked to known disease genes in a network. Interestingly, we show that a combined scoring strategy could enable ICN to achieve an even better performance ( 50\%) than other methods used alone.
\end{abstract}

Conclusions: ICN, a user-friendly method, can well complement other network-based methods in the context of prioritizing candidate disease genes.

\section{Background}

The wide applications of high-throughput techniques have enabled researchers to investigate disease mechanisms in a genome-wide scale $[1,2]$. However, one challenge is that these techniques are usually unable to precisely pinpoint the causative genes. For example, a linkage analysis may give a disease-linked chromosomal region, which may harbor hundreds of candidate genes $[3,4]$; an association study may identify a number of false positives if the disease under investigation has a complex inheritance pattern [5]. While a whole genome re-sequencing can find a number of genetic variations in a patient, only a few of them may play a role in the disease etiology [1]. Therefore, time-consuming and

\footnotetext{
* Correspondence: yang@ym.edu.tw

${ }^{1}$ Institute of Biomedical Informatics, National Yang-Ming University, Taipei

City, Taiwan 11221, Republic of China

Full list of author information is available at the end of the article
}

laborious experiments are usually required to determine the real disease genes from a large number of candidates given by high-throughput experiments. One strategy to accelerate the whole disease gene finding process is to use a computational approach to prioritize candidate genes.

Many computational approaches for prioritizing candidate genes have been developed, assuming that one disease could be caused by a group of functionally related genes. Such approaches measure the functional similarity of each candidate gene to known disease genes using experimentally verified biological data (for details see review [6-9] and Additional File 1). Among these approaches, network-based ones have shown a good performance. The working hypothesis of network-based methods is that genes causing one disease are likely to locate closely to each other in a biological network $[6,10]$. Some network-based methods prioritize

C Biomed Central

(c) 2011 Hsu et al; licensee BioMed Central Ltd. This is an open access article distributed under the terms of the Creative Commons Attribution License (http://creativecommons.org/licenses/by/2.0), which permits unrestricted use, distribution, and reproduction in any medium, provided the original work is properly cited. 
candidate genes based on whether they directly interact with known disease genes [11,12]; other methods further consider the shortest-path distance between candidate genes and known disease genes in a network when direct links do not exist $[13,14]$. On the other hand, different methods might employ distinct scoring strategies. Lage et al. [15] developed a Bayesian predictor that could combine interactome and phenome to infer putative protein complexes likely to associate with a disease. The CIPHER method scores the candidate genes using a regression model of phenotype similarity and gene closeness in a network [16]. Other network-based algorithms, such as random walk [17], network flow [18], page rank [19], network partition [20], and network clustering [21], were also designed to prioritize candidate disease genes.

Network-based methods usually have some parameters that need to be trained using available data sets. The random walk method needs a parameter to control the probability of returning to the initial node [17], and the network flow algorithm uses a parameter to describe the relative importance of prior information [18]. Lage's method requires determining several parameters in order to build the predictor [15]. Whenever biological networks are updated or new training data become available, their parameters should be retuned in order to optimize their performance. It may be difficult for biologists to rep eat these processes by themselves. Additionally, a parameter set may just work for certain cases. Here, we take the random walk (RW) method as an example. Although a parameter setting ( $r=0.5$ ) of RW appears to suffice the identification of many disease genes, using other parameters may be required to find certain disease genes (Figure 1). How to intelligently choose the parameters could be a difficult task to users. We argue that a parameter-

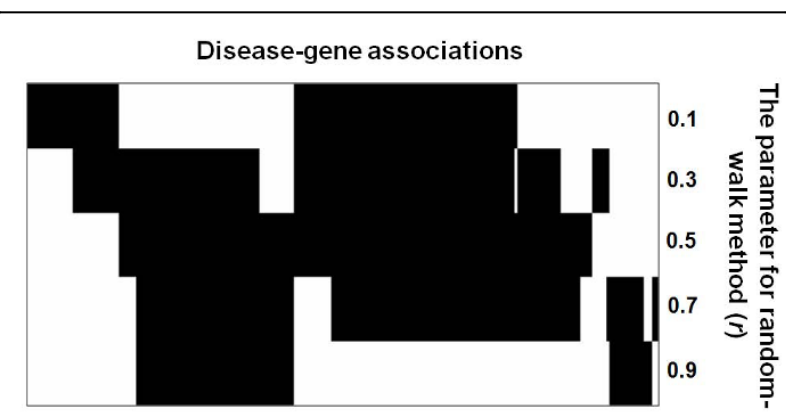

Figure 1 Associations between parameter values and diseasegene association predictions. There are 220 disease-gene association cases in this example. The parameter $r$ is used in random walk method to control the probability of returning to initial node [17]. The solid blocks indicate this method with a specific parameter value successfully gives the true disease genes the highest ranking (for details see the Materials and methods). free algorithm could be more useful to users in this regard.

In this study, we propose a new candidate gene prioritization approach that measures the interconnectedness (ICN) between genes in a network. It was designed to be a parameter-free method. Unlike other networkbased methods, ICN measures closeness of each candidate genes to known disease genes by taking alternative paths into consideration, in addition to the direct link and the shortest-path distance. In comparison with other outperforming network-based methods, ICN is a competitive method. In particular, we show that an impressive performance of prioritizing candidate disease genes could be achieved by combining ICN with other network-based methods. Finally, a novel type of spinocerebellar ataxia (SCA) was chosen to demonstrate the ability of this method.

\section{Results and discussion}

\section{Principles of the interconnectedness-based method}

Most network-based gene prioritization methods, including this one we have developed here, were created on the basis that causative genes of one disease may tend to locate closely in the network $[6,10]$. The approaches taken by various methods differ on how closeness between genes is measured. Before this method is developed, other network-based methods prioritize candidate genes by finding direct-linked disease genes or close disease genes using shortest-path distance. One concern with these previous methods is that they might be less effective than expected if there are noises or missing direct links in the network used to measure inter-gene closeness. Consequently, we designed the InterConnectedNess-based method, ICN, to measure the closeness between genes by considering alternative paths, in addition to the shortest one, that could connect candidate genes to known disease genes. Briefly, ICN determines that these genes are more likely to belong to the same functional module if two genes have more shared interacting genes. A functional module may correspond to a protein complex $[15,18]$ or to a signalling pathway [22]. If a functional module is implicated with a disease, changes to a member gene in this module may cause this disease [23,24]. We applied ICN to the problem of prioritizing disease candidate genes.

\section{Comparison with other network-based prioritization algorithms}

According to the comprehensive comparison performed in [25], the best two outperforming methods for prioritizing candidate genes were the Random Walk method (RW) [17] and the PRINCE (PRIoritizatioN and Complex Elucidation) algorithm (PR) [18]. In this project, they were re-implemented in order to compare their 
performance with that of ICN. Their parameters were optimized as described in [18] (for details see Materials and Methods).

Two biological networks were recruited as the data sets to evaluate the performance of ICN and other two methods. These networks were chosen because each network has features distinctive from that of the other. We intended to examine if each method could perform in a consistent manner using different types of network data. The first one is a protein-protein interaction network (PIN) consisting of 140,382 interactions and 12,164 genes. PIN consists of data retrieved from nine protein-protein interaction data sources [26-34]. The second one is a functional association network (FAN) consisting of 1,217,908 interactions and 16,648 genes downloaded from the STRING database [35]. These two networks share 11,776 common genes and 95,630 common interactions. Two major differences between these data sets are the number of interactions and the types of edges. While PIN edges are un-weighted, FAN edges are annotated with weights indicating the confidence of functional linkage between each pair of connected genes [36]. ICN is able to incorporate edge weights in quantifying the closeness between genes in a network. The statistics of available data in each network is summarized in Table 1.

A leave-one-out procedure was employed to carry out the evaluation. The disease-gene associations were obtained from OMIM [37]. These genes were manually grouped in to different disease families as described in Materials and Methods. In each validation trial, the association of one test gene with a disease family was removed, and each method was tried to re-build this association. To mimic the situations we may encounter when using different high-throughput genome-wide techniques to find disease genes, we created two test scenarios, the simulated linkage analysis and the whole genome scan. In the simulated linkage analysis, each time a test disease gene together with 100 genes on its flanking regions was taken as the candidate set. In the whole genome scan, each time a test disease gene together with all human genes in the network, excluding other members from the corresponding disease family, was taken as the candidate set. If a test gene was ranked top $k$ in a candidate set in a trial, this trial was regarded as a successful one. We further defined the "success rate" as the fraction of successful trials for a method under a particular test scenario.

The results of simulated linkage analysis for each method are presented in Figure 2. 1,993 and 2,616 disease-gene associations were tested using PIN and FAN, respectively. When PIN was used, ICN achieved the best performance with a success rate of $44.7 \%$, ranking the known disease genes as top 1 candidate $(k=1)$ in 870 out of 1,993 cases. RW and PR also achieved the similar performance with a success rate of $43.3 \%(862 / 1993)$ and $43.4 \%$ (865/1993), respectively. When the rank cutoff $(k)$ was increased, PR had the best performance, while the performance of ICN was still comparable with that of PR (Figure 2A). When FAN was used, RW achieved a success rate of $71.3 \%(1865 / 2616)$, better than that ICN $(64.1 \%, 1678 / 2616)$ and PR $(66.4 \%, 1738 /$ 2616) did. On the other hand, as rank cutoff was increased $(k>=5)$, the performance of ICN and PR was better than that of RW (Figure 2B).

The performance comparison under the test scenario of whole genome scan is shown in Figure 3. When PIN was used, ICN successfully ranked the known disease genes as top 1 candidate in 192 out of 1,993 cases, with a success rate of 0.096 . RW performance with a success rate of $15.0 \%(299 / 1,993)$ was higher than ICN and PR $(6.9 \%, 137 / 1,993)$. Similarly, the performance of ICN $(10.4 \%, 272 / 2,616)$ was between RW $(19.1 \%, 499 / 2,616)$ and PR $(6.7 \%, 174 / 2,616)$ when FAN was used. The benchmark reveals that although ICN did not outperform in all cases, it was quite comparable to other methods.

If the cases with disease genes being ranked as top 1 candidates by at least one of three prioritization methods were considered as successful predictions, the overall success rates so achieved were $54.3 \%(1,083 / 1,993)$ by using PIN and $79.2 \%(2,073 / 2,616)$ by using FAN,

Table 1 Statistics of biological networks

\begin{tabular}{lll}
\hline & Protein-protein interaction network (PIN) & $\begin{array}{c}\text { Functional association network } \\
(\text { FAN) }\end{array}$ \\
\hline Data source(s) & $\begin{array}{l}\text { Integration from DIP, BOND, IntAct, MINT, MIPS, HPRD, BioGRID, Reactome, and pathway } \\
\text { commons } \\
\text { UntRING v8.2 }\end{array}$ \\
Network type & 12,164 & Weighted \\
\# genes & 140,382 & 16,648 \\
\# interactions & 344 & $1,217,908$ \\
\# disease families & 1,993 & 509 \\
\# disease-gene & 1,640 & 2,616 \\
associations & & 1,909 \\
\# disease genes & &
\end{tabular}


(A)

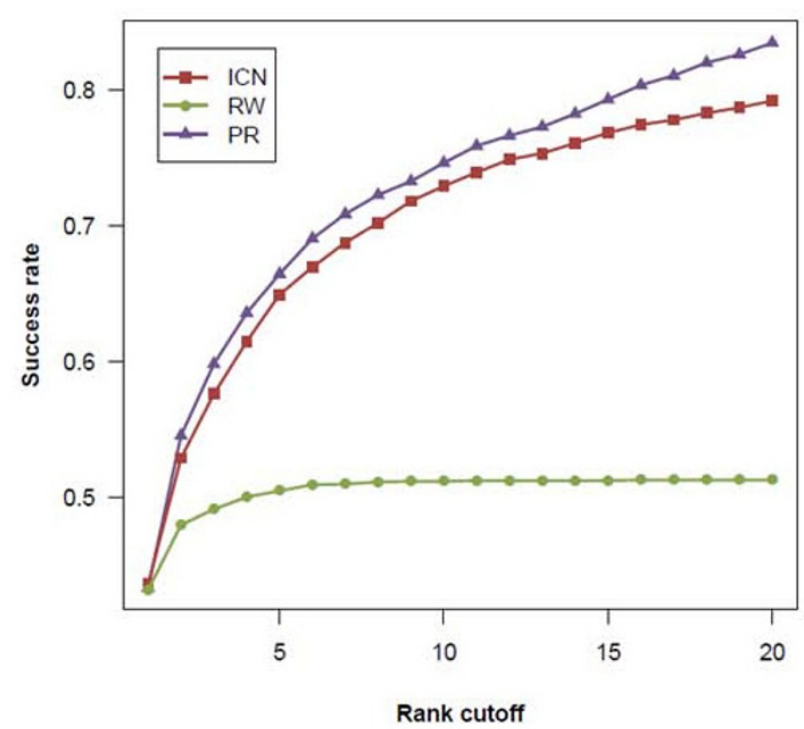

(B)

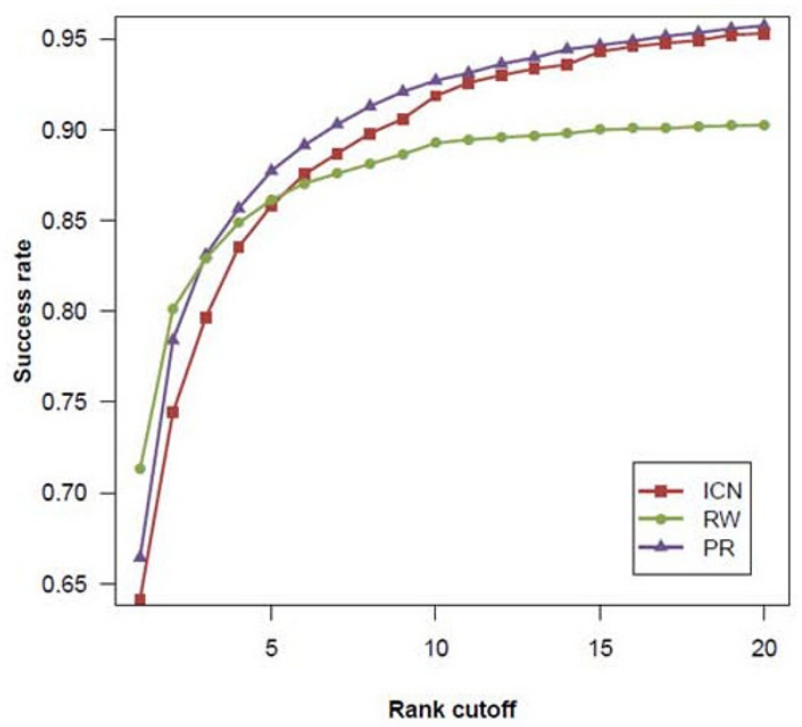

Figure 2 A performance of prioritization methods tested on simulated linkage analyses. The performance of different methods is assessed by using PIN (A) and FAN (B), respectively.

respectively, under the test scenario of simulated linkage analysis. The overall performance was much better than that of respective methods. Figure 4 presents the overlaps of successful predictions among ICN, RW, and PR. No matter which biological network was used, RW and PR shared more success cases than other combinations. This is not really surprising, since RW and PR took a similar iterative procedure to look for candidate genes in a network $[17,18]$. Interestingly, each method predicted unique cases. In particular, ICN gave the highest number of unique success cases using PIN, and it gave a comparable number of unique cases with that of RW using FAN. These results indicate that each method may perform better than other methods on certain cases. Analyzing the difference of the unique success cases generated by different methods may help us get a deeper understanding of unique advantage of each method, which could assist us to further improve the performance.

\section{Exploring the cases uniquely predicted by respective methods}

Intuitively, topological properties of genes in a network may affect the performance of candidate gene prioritization when network-based methods are used. To understand how the performance of different methods could be influenced, we examined if the disease genes uniquely identified by individual methods had distinctive topological properties. For simplicity, disease genes uniquely identified by ICN are denoted as ICN-unique genes/ cases, and so forth for other methods, in the following text.

Firstly, the number of interacting partners, also referred to as the degree in the graph theory [38], of each method-unique case was considered. We noticed that when PIN was used, the average degree of RWunique cases was significantly higher than these of ICNand PR-unique cases ( $\mathrm{P}$-value $=0.002$ and $2.9 \times 10-6$, Wilcoxon signed-rank test). Secondly, we explored to which extent a method-unique gene may be located, in a network, away from the known genes implicated in a disease family. Here we found that when PIN was used, the distribution of the shortest-path distances of ICNunique cases is similar to that of PR-unique cases (Figure $5 \mathrm{~B}$ ). Both $\mathrm{ICN}$-unique and PR-unique cases are significantly more distant from known disease genes than that of RW-unique cases $(\mathrm{P}$-values $=1.9 \times 10-5$ and $2.6 \times 10-5$, respectively, Wilcoxon signed-rank test). The analysis of the method-unique cases using FAN yielded a similar result (Additional File 2).

On the whole, these results support that a prioritization method may outperform the others when candidate disease genes to be assessed have certain method-favored topological properties. When candidate genes have more interacting partners in a network and are closer to other known disease genes, RW may perform better than the other methods. In contrast, ICN and PR may outperform RW when 


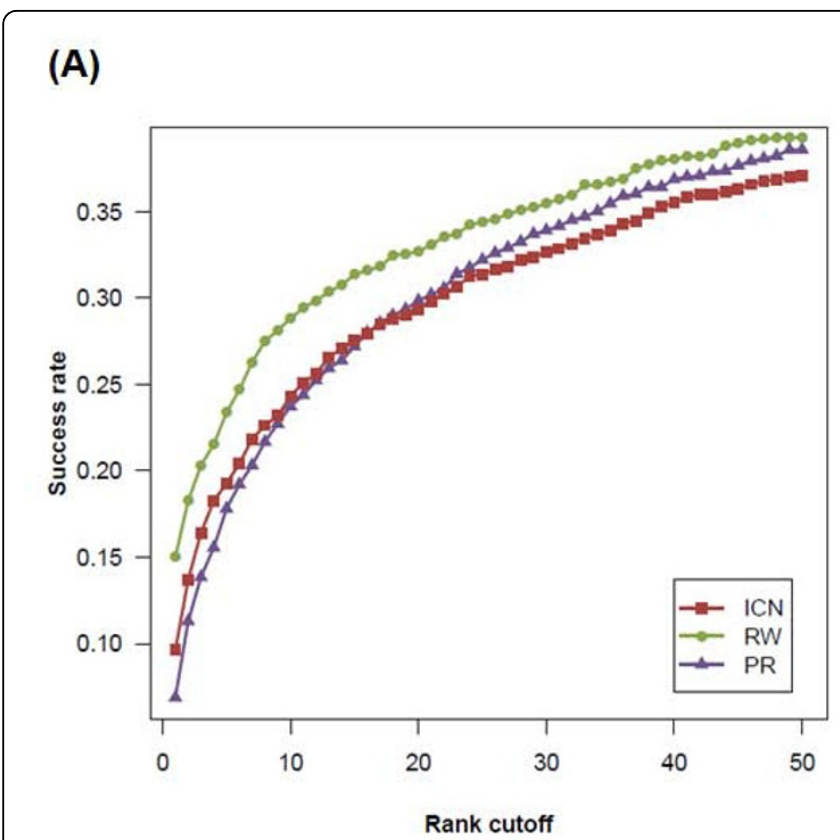

(B)

Figure $3 \mathrm{~A}$ performance of prioritization methods tested on whole genome scans. The performance of different methods is assessed by using PIN (A) and FAN (B), respectively.

prioritizing candidate genes that are more distant away from other known disease genes in a network. Therefore, it is quite possible that combining the ranking results of different methods may further improve the performance of candidate gene prioritization. In the next section, we show that a combined scoring strategy did improve the performance of prioritizing candidate disease genes.
(A)

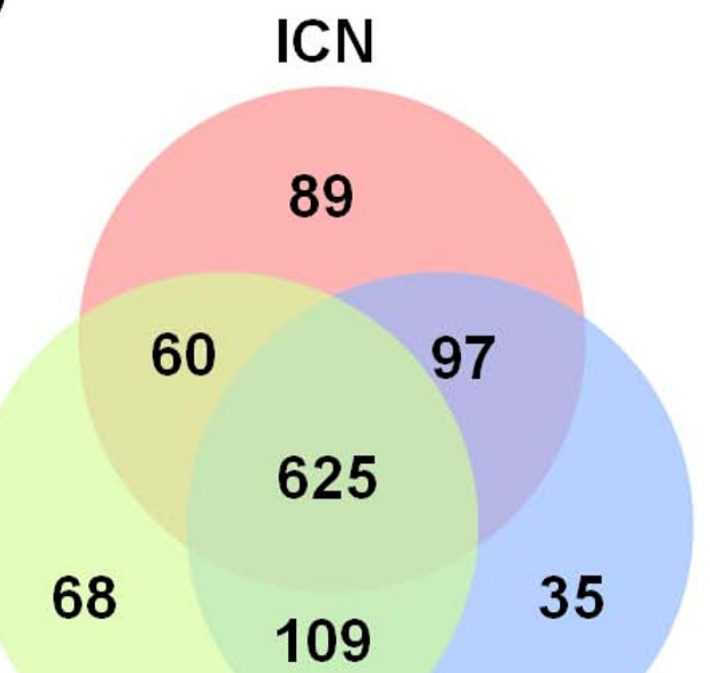

RW

Figure 4 Venn diagram of successfully predicted cases among different prioritization methods. The cases which are successfully ranked the known disease genes as top 1 candidate are compared among ICN, RW, and PR by using PIN (A) and FAN (B), respectively.
(B)

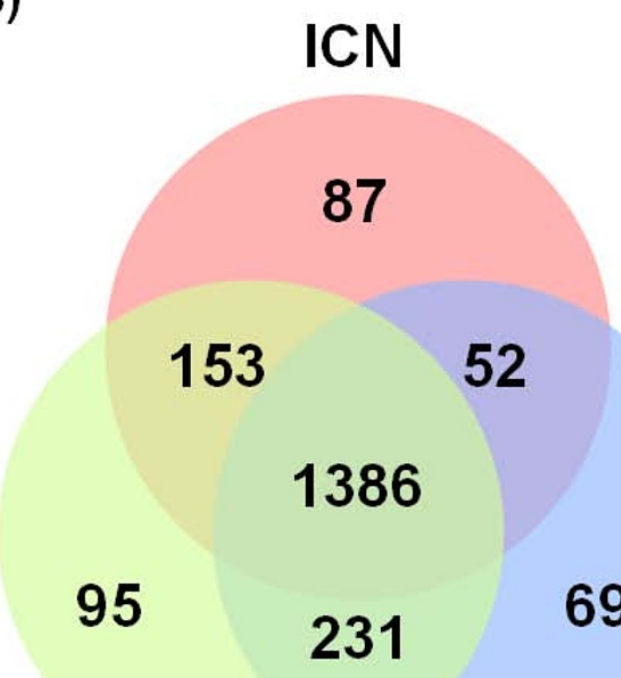

RW 


\section{Improving the performance using a combined scoring strategy}

Since each method may have its own favorite cases, we tried to improve the performance of prioritization by combining the results generated by different methods.

To preserve the unique advantage of each method, we did not change any algorithmic approaches in them. Instead, we used a combined scoring strategy by multiplying together the ranks generated by different methods (for details see section Materials and Methods). The performance of this new approach was also evaluated using the leave-one-out procedure under a test scenario of either simulated linkage analysis and whole-genome scan.

Table 2 lists the performances of respective methods and different combined scoring schemes tested in the simulated linkage scenario. Here, we denote the scoring scheme of combining the ranking results of ICN and PR as the ICN-PR method, and so forth. Interestingly, all combined scoring schemes achieved higher success rates than respective methods. When PIN was used, the ICNPR method showed the best performance (success rate 48.9\%). Besides, the ICN-RW method also showed a better success rate $(46.9 \%)$ than respective methods. On the other hand, when FAN was used, the RW-PR method outperformed the other individual and combined methods (success rate $73.7 \%$ ). The ICN-PR method achieved a success rate $(72.7 \%)$ close to the best one. All the combined scoring schemes made substantial performance improvement compared to respective methods
Table 2 Success rates of ranking known disease genes as the best candidate

\begin{tabular}{llllllll}
\hline $\begin{array}{l}\text { Success rate } \\
\text { (\%) }\end{array}$ & ICN & RW & PR & $\begin{array}{l}\text { ICN- } \\
\text { RW }\end{array}$ & $\begin{array}{l}\text { ICN- } \\
\text { PR }\end{array}$ & $\begin{array}{l}\text { RW- } \\
\text { PR }\end{array}$ & $\begin{array}{l}\text { ICN-RW- } \\
\text { PR }\end{array}$ \\
\hline PIN & 43.7 & 43.3 & 43.4 & 46.9 & 48.9 & 46.8 & 44.5 \\
FAN & 64.1 & 71.3 & 66.4 & 72.7 & 73.3 & 73.7 & 72.4 \\
\hline
\end{tabular}

(ICN: 64.1\%, RW: 71.3\%, PR: 66.4\%). Finally, when these combined scoring schemes were tested in the whole genome scan scenario, no performance improvement could be found (data not shown). It is not surprising since we expect that there could be missing parts in currently available biological networks and more genes are yet to be identified to fill in the networks.

Here we further explored if the cases failed when respective methods were used could be recovered using the combined scoring schemes. The result is listed in Table 3. When PIN was used, 11 and 25 cases (out of 911 cases failed using respective methods) could be recovered by the ICN-RW and the ICN-PR methods, respectively, but no cases could be recovered by the RW-PR method or the ICN-RW-PR method. We also tested if it could make a difference if FAN was used. It turned out that the ICN-RW method and the ICN-PR method rescued 27 and 22 cases (out of 543 cases failed using respective methods), respectively. The RW-PR method could rescue only one case, and the ICN-RW -PR method did not really show a much better performance (4 cases rescued).
(A)

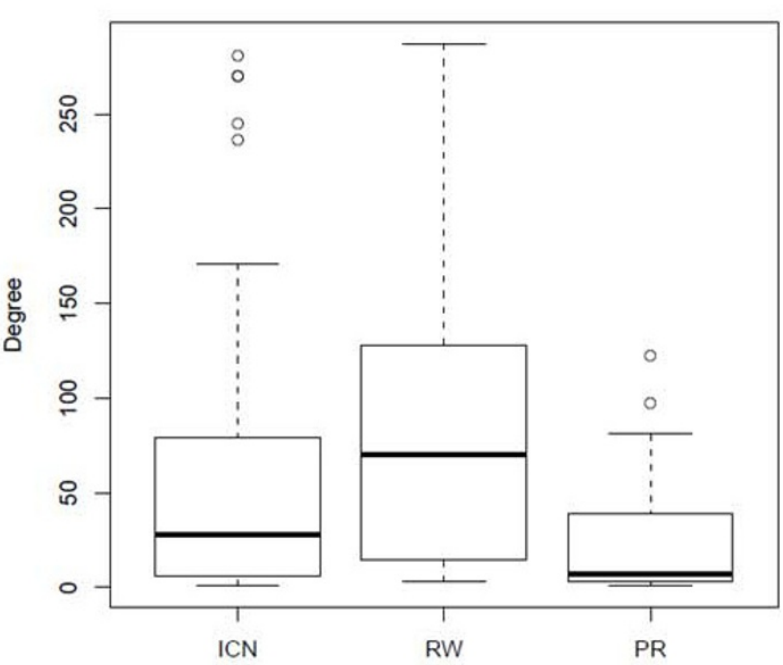

(B)

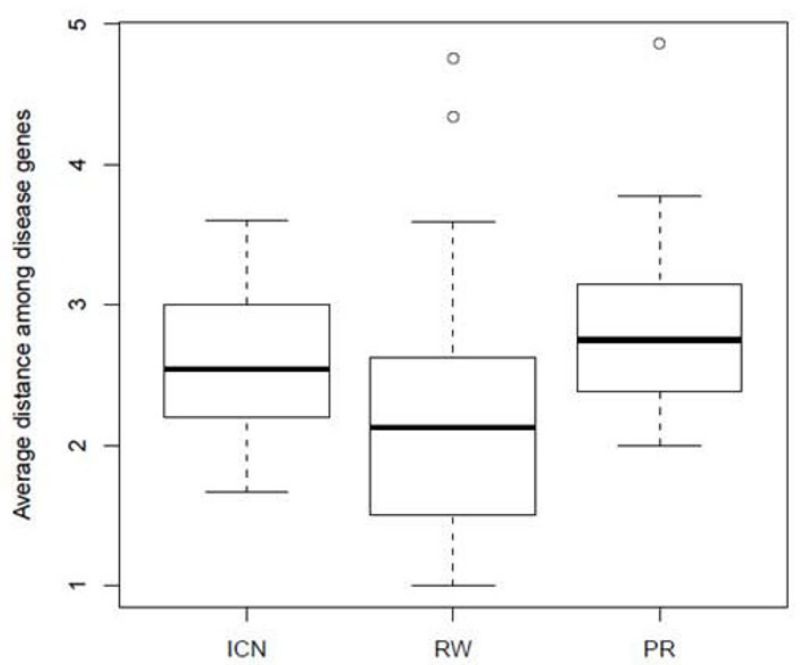

Figure 5 Analysis of network topological properties on disease causing genes. The topological properties of disease genes in unique cases which were successfully ranked the known disease genes as top 1 candidate by a specific method in PIN (Figure 3A) were compared in degree (A) and average shortest-path distance between other disease-associated genes which are in the same disease family(B). 
Table 3 Failed prediction cases recovered by combined methods

\begin{tabular}{llllll}
\hline \multirow{2}{*}{ \# failed prediction cases $^{\&}$} & \multicolumn{4}{l}{ \# cases re-ranked as top 1 candidate } \\
\cline { 3 - 6 } & & ICN-RW & ICN-PR & RW-PR & ICN-RW-PR \\
\hline PIN & 911 & 11 & 25 & 0 & 0 \\
FAN 543 & 27 & 22 & 1 & 4 \\
\hline
\end{tabular}

\& a failed prediction case indicates that no prioritization method can rank the true disease gene as top 1 candidate.

All in all, combining the results of different networkbased methods indeed enhances the performance of prioritizing candidate disease genes. In particular, substantial performance improvement was made when combining ICN with other methods.

\section{Using ICN and combined scoring schemes to find spinocerebellar ataxia genes}

To demonstrate the ability of ICN and the combined scoring schemes in finding novel disease genes, we present a case study for spinocerebellar ataxia type 22 (SCA22) [39]. Autosomal dominant spinocerebellar ataxias (SCAs) are a group of progressive neurodegenerative disorders characterized by the loss of balance and motor coordination due to dysfunction of the cerebellum [40]. SCAs are genetically heterogeneous. To date, more than 30 genomic loci have been linked to different subtypes of SCA; however, only 18 causative genes have been determined $[41,42]$. Interestingly, these genes share common interacting partners [43], suggesting that network-based methods could be suitable for finding novel SCA-causing genes. SCA22 has been found to link to the locus on chromosome 1q21-23 [39], where 541 protein-coding genes were annotated (Ensembl release 58, http://www.ensembl.org). Our aim was to prioritize these 541 candidate disease genes.

The confirmed SCA-causing genes in Table 4 were regarded as known disease genes for the SCA disease family. There were 15 and 17 of them in PIN and FAN, respectively. Table 5 and 6 present the top 10 candidate genes (i.e. $k=10$ ) prioritized using PIN and FAN, respectively. Firstly, we tested individual methods. We noticed that ICN, RW, and PR generated very different results. No identical top one gene could be consistently determined by different methods. In addition, when PIN was used, only 2 genes, SPTA1 and GNAT2, were commonly identified by all methods $(k=10$, Table 5$)$. Similarly when FAN was used, only 3 genes (KCNN3, SPTA1, and KCNC4) commonly identified by all methods $(k=10$, Table 6$)$.

Secondly, we tested combined scoring schemes and they appeared to generate more consistent results. When PIN and FAN were used respectively, there were correspondingly three (SPTA1, GNAT2, and NRAS) and
Table 4 List of SCA-causing genes

\begin{tabular}{|c|c|c|c|}
\hline SCA subtype & Gene & $\mathrm{PIN}^{\&}$ & $\mathrm{FAN}^{8}$ \\
\hline$\overline{\text { SCA1 }}$ & ATXN1 & $Y$ & $Y$ \\
\hline SCA2 & ATXN2 & Y & Y \\
\hline SCA3 & ATXN3 & Y & Y \\
\hline SCA5 & SPTBN2 & Y & Y \\
\hline SCA6 & CACNA1A & Y & Y \\
\hline SCA7 & ATXN7 & Y & Y \\
\hline SCA8 & ATXN8 & $\mathrm{N}$ & $\mathrm{N}$ \\
\hline SCA10 & ATXN10 & Y & Y \\
\hline SCA11 & TTBK2 & Y & Y \\
\hline SCA12 & PPP2R2B & Y & Y \\
\hline SCA13 & KCNC3 & $\mathrm{N}$ & Y \\
\hline SCA14 & PRKCG & Y & Y \\
\hline SCA15 & ITPR1 & Y & Y \\
\hline SCA17 & TBP & Y & Y \\
\hline SCA27 & FGF14 & $\mathrm{N}$ & Y \\
\hline SCA28 & AFG3L2 & Y & Y \\
\hline SCA31 & PLEKHG4 & Y & Y \\
\hline DRPLA & ATN1 & Y & Y \\
\hline
\end{tabular}

\& whether the disease genes are in the given network. DRPLA: dentatorubralpallidoluysian atrophy

seven (KCNN3, SPTA1, CCT3, KCNC4, KCNA2, KCND3, and KCNA3) common genes identified by all combined scoring schemes $(k=10$, Table 5 and 6$)$. Furthermore, SPTA1 and KCNN3 were consistently picked out as the best candidates by all combined scoring schemes using PIN and FAN, respectively. SPTA1 was also ranked in the top 3 candidate genes by combined scoring schemes when FAN was used. KCNN3 was not included in the candidate list when PIN was used because there was no interaction information for KCNN3.

From protein function and literature survey, we found that SPTA1 and KCNN3 are very likely to associate with SCA22. SPTA1 is a member of spectrin family, functioning in actin crosslinking and as the molecular scaffold proteins to determine cell shapes and to arrange the transmembrane proteins. An in-frame deletion in

Table 5 Top 10 candidate genes for SCA22 by using PIN

\begin{tabular}{llllllll}
\hline Rank & ICN & RW & PR & ICN-RW & ICN-PR & RW-PR & $\begin{array}{l}\text { ICN-RW- } \\
\text { PR }\end{array}$ \\
\hline 1 & SPTA1 & NRAS & YY1AP1 & SPTA1 & SPTA1 & SPTA1 & SPTA1 \\
2 & GNAT2 & SPTA1 & ECM1 & GNAT2 & YY1AP1 & AHCYL1 & GNAT2 \\
3 & TAF13 & GNAT2 & AHCYL1 & NRAS & GNAT2 & NRAS & NRAS \\
4 & ISG20L2 & GNAI3 & FDPS & ISG20L2 & TAF13 & ECM1 & YY1AP1 \\
5 & FCGR2C & AHCY1 & SPTA1 & FCGR2C & ECM1 & YY1AP1 & ECM1 \\
6 & YY1AP1 & STXBP3 & STXBP3 & TAF13 & NRAS & GNAT2 & TAF13 \\
7 & PSMD4 & ECM1 & S100A7 & PSMD4 & STXBP3 & STXBP3 & STXBP3 \\
8 & NRAS & CCT3 & POLR3C & GNAI3 & PSMD4 & GNAI3 & AHCYL1 \\
9 & NGF & RPS27 & UBAP2L & NGF & POLR3C & S100A7 & GNAI3 \\
10 & NTRK1 & S100A7 & GNAT2 & NTRK1 & NGF & FDPS & PSMD4 \\
\hline
\end{tabular}


Table 6 Top 10 candidate genes for SCA22 by using FAN

\begin{tabular}{llllllll}
\hline Rank & ICN & RW & PR & ICN-RW & ICN-PR & RW-PR & ICN-RW-PR \\
\hline 1 & S100A6 & SPTA1 & KCNN3 & KCNN3 & KCNN3 & KCNN3 & KCNN3 \\
2 & KCNN3 & CCT3 & HCN3 & SPTA1 & S100A6 & SPTA1 & SPTA1 \\
3 & NGF & KCNN3 & SPTA1 & S100A6 & SPTA1 & CCT3 & S100A6 \\
4 & KCNA2 & S100A11 & PPM1J & KCNA2 & KCNC4 & HCN3 & CCT3 \\
5 & KCNC4 & KCNA2 & RHBG & CCT3 & PPM1J & KCNC4 & KCNC4 \\
6 & KCND3 & KCNA3 & KCNC4 & KCNC4 & KCNA2 & KCNA2 & KCNA2 \\
7 & KCNA3 & KCND3 & AHCYL1 & KCND3 & KCND3 & S100A11 & KCND3 \\
8 & SPTA1 & KCNC4 & CCT3 & KCNA3 & CCT3 & PPM1J & KCNA3 \\
9 & HIST2H2BE & ARHGEF11 & PYGO2 & NGF & KCNA3 & KCNA3 & PPM1J \\
10 & SHC1 & CD5L & F11R & F11R & F11R & KCND3 & F11R \\
\hline
\end{tabular}

SPTBN2, which is also a member of the spectrin family, can cause SCA5 [44]. Recent studies have shown that the mutant SPTBN2 disrupts fundamental intracellular transport processes in synapses [45-47]. This is likely to contribute to progressive neurodegenerative disease, such as SCA. Therefore, SPTA1 may cause SCA22 in a similar mechanism. Besides, KCNN3 is a member of the gene family encoding the small conductance calciumactivated potassium channels. A CAG repeat polymorphism has been annotated in the amino-terminal coding region of KCNN3 [48]. Many studies revealed that such repeat polymorphisms associate with psychiatric diseases, such as schizophrenia [49] and bipolar diseases [50].

To further validate these two candidates experimentally, an exome sequencing experiment was performed, and several novel gene variations have been found on SPTA1 in two SCA22 patients (Chung, M.-Y. et al., unpublished data). This preliminary result we present here suggests that ICN and the combined scoring schemes are able to identify the novel disease genes.

\section{Conclusions}

The InterConnectedNess-based method (ICN) is a biologically intuitive and parameter-free approach for prioritizing candidate disease genes. There is no need for users to train the parameters every time when biological networks to be used are updated. ICN not only was comparable to other well-known methods, such the random walk method (RW) and the PRINCE algorithm (PR), but also outperformed these methods when candidate disease genes are located more distantly to known disease genes in a network. Furthermore, combined ICN-RW or ICN-PR scoring schemes showed an impressive performance improvement in prioritizing candidate disease genes, suggesting that different network-based methods may complement the weakness of each other.

In this study, we created a very simple combined scoring strategy by multiplying the ranks generated by different methods. The success of this strategy implies that there might still be a chance to further improve the performance of network-based methods in prioritizing candidate disease genes. To achieve this, we plan to try other strategies. In addition to combining method-specific ranking results, combining network-specific ranking results appears to be another promising strategy. In fact, two algorithms, $\mathrm{N}$-dimensional order statistics (NDOS) [51] and discounted rating system (DRS) [52], have been employed in some prioritization methods to combine ranking results generated respectively by using different network data sets. It would be interesting to find out if the performances of ICN or other network-based methods can still be advanced when more heterogeneous approaches are integrated together.

\section{Materials and methods Biological networks}

Two kinds of biological networks were employed to test the performance of network-based methods in this study: protein-protein interaction network (PIN) and functional association network (FAN). PIN was constructed by integrating protein-protein interaction data from nine databases, including DIP [26], BIND [27], IntAct [28], MIPS [29], MINT [30], HPRD [31], BioGRID [32], Reactome [33], and Pathway Commons [34]. Another dataset, FAN, was obtained from STRING v8.2, which was a comprehensive gene association dataset containing directly physical interactions and functional links from experimental evidence and computational methods [35]. In both networks, the identifier for each gene was mapped to Entrez Gene ID, and self-interacting pairs were removed. Finally, PIN consists with 140,382 interactions and 12,164 genes, and FAN consists of 1,217,908 interactions and 16,648 genes (Table 1). Each connection in FAN was assigned a confidence scores from STRING, which reflects the confidence of each gene-gene association. PIN and FAN were regarded as unweighted and weighted networks, respectively. 


\section{Disease-gene associations}

The disease-gene associations were retrieved from the Morbid Map in OMIM [37]. If the causative genes were not included in the networks, their associations to diseases were removed. Because the prioritizing methods require related disease genes for prediction, the related causative genes were manually grouped into a disease family based on their given disorder name [53], and disease families that have only one causative gene were filtered out. In total, 1,993 disease-gene associations implicated with 344 disease families were recruited in PIN and 2,616 disease-gene associations implicated with 509 disease families were recruited in FAN (Table 1).

\section{Interconnectedness (ICN) between genes}

The closeness between genes in a network was quantified by considering not only direct interaction of two genes but also the number of connectors between genes. As illustrated in Figure 6, the interconnectedness score $I C N_{i, j}$ between two genes $i$ and $j$ was defined as:

$$
I C N_{i, j}=\frac{2 * \omega_{i, j}+\sum_{u \in\left(N_{i} \cap N_{j}\right)} \omega_{i, u} \omega_{j, u}}{\sqrt{k_{i} k_{j}}}
$$

where $N$ is the neighboring genes of a given gene, and $u$ is the gene linked to both gene $i$ and $j . \omega$ is a weight of the connection between two genes, e.g. $\omega_{i, j}$ corresponds to the weight between gene $i$ and $j$. In FAN, the value of $\omega$ is within the interval between 0 and 1 . In PIN, however, $\omega$ is either 1 or 0 , i.e. connected or unconnected. Because the number of connectors may be associated with the number of neighbors of each node, the number of connectors between two genes is normalized by the expected number of connectors between these genes. $k_{i}$ is the sum of weights of gene $i$ 's neighboring connections and is defined as:

$$
k_{i}=\sum_{j \in N_{i}} \omega_{i, j}
$$

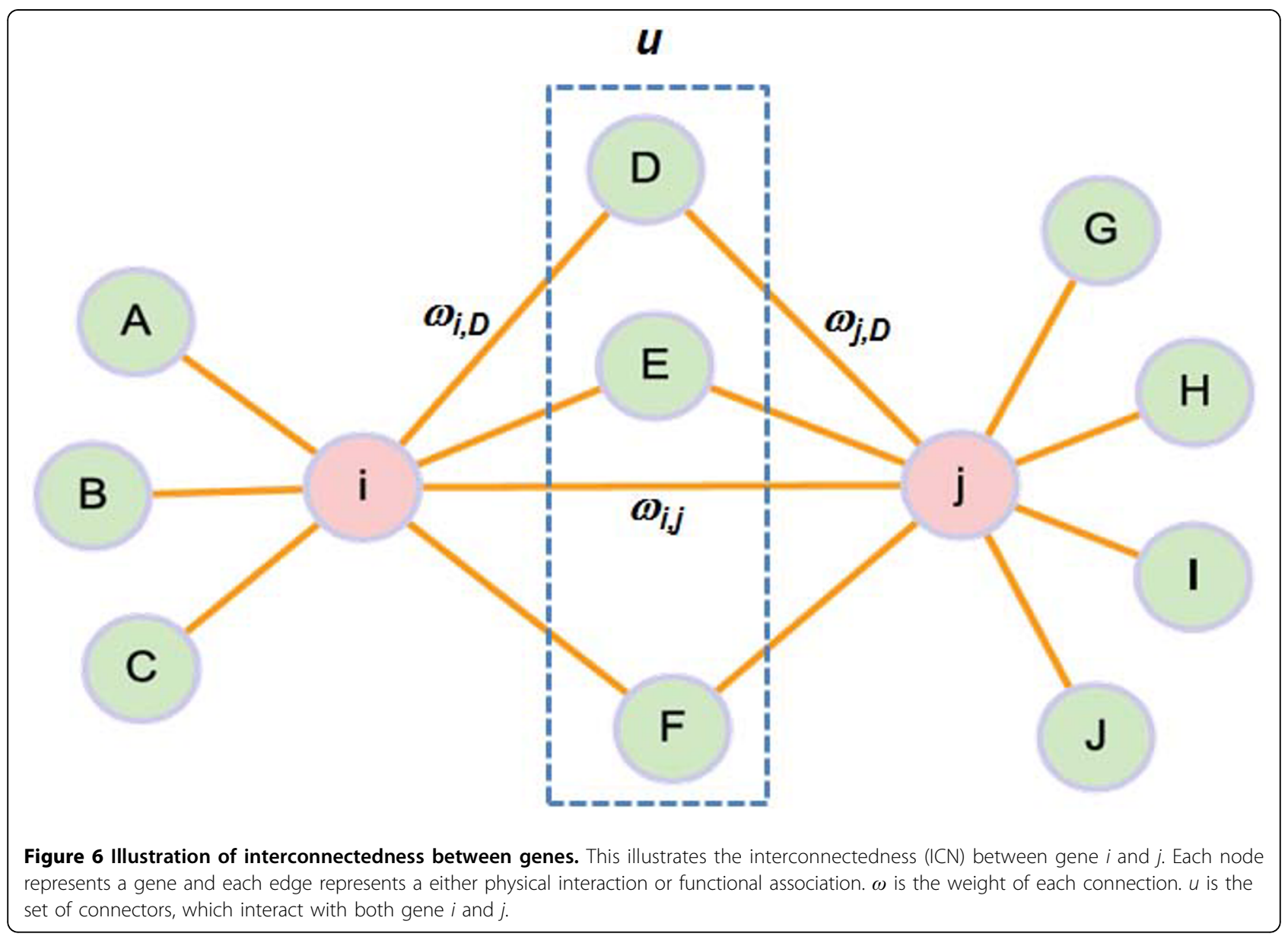


In an unweighted network, $k_{i}$ corresponds directly to the degree, namely the number of neighbors of a given gene [38].

Prioritizing candidate genes by interconnectedness scores Candidate genes are then prioritized based on the ICN scores calculated using equation 1 . For a given disease $d$, each candidate gene was scored by summing up the closeness to the seed genes $S_{d}$, i.e. the genes in the same disease family. The score of a given candidate gene $i$ was calculated as:

$$
\text { score }_{i}=\frac{1}{\left|S_{d}\right|} \sum_{j \in S_{d}} \operatorname{ICN}_{i, j}
$$

where $I C N_{i, j}$ is the connection score between gene $i$ and $j$. All candidate genes are then ranked based on these scores.

\section{Implement of random walk (RW) and PRINCE (PR) methods}

Both the random walk (RW) method [17] and the PRINE (PR) algorithm [18] apply an iterative procedure to find candidate disease genes in a network. When the difference between results of the previous and current steps (measured by $L_{1}$-norm) fell below $10^{-10}$, the iteration was halted, and candidate genes were ranked based on the scores in the final step.

The precise behaviors employed by the two methods to reach candidate genes in a network differ. RW [17] simulates a random walker that starts from one or a set of source nodes, and moves forward to neighboring nodes with a probability proportional to the weight of the connecting edge. RW also allows the walker to move back to the source node with probability $r$ in each step. $r$ controls how far the random walker could get away from the source node. PR [18], a propagationbased algorithm, exploits prior information on causative genes for the same disease or similar ones and infers a strength-of-association function to smooth over the network (i.e. adjacent nodes are assigned similar values). The parameter $\alpha$ in PR controls the relative importance of prior information. Using the tuning procedure described in [18], we set $r=0.5$ and $\alpha=0.9$, which make corresponding methods achieve the optimal performance when the two network data sets described in this study are used.

\section{Experiment design and performance measurement}

Two test scenarios were designed to evaluate the performance of all methods: simulated linkage analyses and whole genome scan. In the simulated linkage analysis, a total of 100 genes flanking a test disease gene were taken as the candidate genes. In the whole genome scan, a test disease gene and all the genes in a biological network excluding other members from the corresponding disease gene family constitute the candidate gene list.

A leave-one-out procedure is used to assess the performance of the different methods. In each trial, a disease-gene association was removed and remaining genes in the same disease family were taken as seed genes to reconstruct the association. We used the "success rate" to represent the performance of a method. If the removed disease-gene association was ranked in top $k$ of a candidate gene list, this trial was regarded as a successful prediction. The "success rate" of a method is defined as the fraction of successful predictions in all cases tested given a particular combination of a network data set and a test scenario.

\section{Combing the prioritization results given by different methods}

For each candidate gene $i$, a combined score $C S_{i}$ was calculated as:

$$
C S_{i}=\prod_{j=1}^{n} R_{i, j}
$$

where $R_{i, j}$ indicates the rank of gene $i$ in method $j$. The candidate genes were re-ranked using the combined scores in an ascending order, i.e. the lower combined score, the higher priority.

\section{Additional material}

Additional file 1: List of related algorithms and tools for prioritizing disease candidate genes

Additional file 2: Analysis of network topological properties on disease causing genes The topological properties of disease genes in unique cases which were successfully ranked the known disease genes as top 1 candidate by a specific method in FAN (Figure 3B) were

compared in degree (A) and average shortest-path distance between other disease-associated genes which are in the same disease family(B).

\section{List of abbreviations used}

FAN: functional association network; ICN: interconnectedness; OMIM: Online Mendelian Inheritance in Man; RW: random work method; PIN: proteinprotein interaction network; PR: PRINCE algorithm.

\section{Acknowledgements}

We would like to thank the anonymous referees for helpful comments on this paper. This work was supported by the National Research Program in Genomic Medicine (NRPGM), the National Science Council (NSC100-2319-B010-002), Ministry of Education, Aiming for the Top University Plan, and National Yang-Ming University, Taiwan.

This article has been published as part of BMC Genomics Volume 12 Supplement 3, 2011: Tenth International Conference on Bioinformatics - First ISCB Asia Joint Conference 2011 (InCoB/ISCB-Asia 2011): Computational Biology. The full contents of the supplement are available online at http:// www.biomedcentral.com/1471-2164/12?issue=S3. 


\section{Author details}

${ }^{1}$ Institute of Biomedical Informatics, National Yang-Ming University, Taipei City, Taiwan 11221, Republic of China. ${ }^{2}$ Department of Biochemistry, Faculty of Medicine, School of Medicine, National Yang-Ming University, Taipei City, Taiwan 11221, Republic of China. ${ }^{3}$ Center for Systems and Synthetic Biology, National Yang-Ming University, Taipei City, Taiwan 11221, Republic of China.

\section{Authors' contributions}

CLH conceived and designed the experiments. CLH and CTH performed the experiments. CLH, YHH and UCY analyzed and interpreted data. All the authors wrote and revised the manuscript.

\section{Competing interests}

The authors declare that they have no competing interests.

Published: 30 November 2011

\section{References}

1. Kuhlenbaumer G, Hullmann J, Appenzeller S: Novel genomic techniques open new avenues in the analysis of monogenic disorders. Hum Mutat 2011, 32(2):144-151.

2. Tang WC, Yap MK, Yip SP: A review of current approaches to identifying human genes involved in myopia. Clin Exp Optom 2008, 91(1):4-22.

3. Botstein $D$, Risch $N$ : Discovering genotypes underlying human phenotypes: past successes for mendelian disease, future approaches for complex disease. Nat Genet 2003, 33(Suppl):228-237.

4. Glazier AM, Nadeau JH, Aitman TJ: Finding genes that underlie complex traits. Science 2002, 298(5602):2345-2349.

5. McCarthy MI, Abecasis GR, Cardon LR, Goldstein DB, Little J, loannidis JP, Hirschhorn JN: Genome-wide association studies for complex traits: consensus, uncertainty and challenges. Nat Rev Genet 2008, 9(5):356-369.

6. Oti M, Brunner HG: The modular nature of genetic diseases. Clin Genet 2007, 71(1):1-11.

7. Zhu M, Zhao S: Candidate gene identification approach: progress and challenges. Int J Biol Sci 2007, 3(7):420-427.

8. Kann MG: Advances in translational bioinformatics: computational approaches for the hunting of disease genes. Brief Bioinform 2010 11(1):96-110.

9. Tranchevent LC, Capdevila FB, Nitsch D, De Moor B, De Causmaecker P, Moreau $Y$ : A guide to web tools to prioritize candidate genes. Brief Bioinform 2011, 12(1):22-32

10. Ideker T, Sharan R: Protein networks in disease. Genome Res 2008, 18(4):644-652

11. Chen JY, Shen C, Sivachenko AY: Mining Alzheimer disease relevant proteins from integrated protein interactome data. Pac Symp Biocomput 2006, 367-378

12. Oti M, Snel B, Huynen MA, Brunner HG: Predicting disease genes using protein-protein interactions. J Med Genet 2006, 43(8):691-698.

13. Krauthammer M, Kaufmann CA, Gilliam TC, Rzhetsky A: Molecular triangulation: bridging linkage and molecular-network information for identifying candidate genes in Alzheimer's disease. Proc Natl Acad Sci U S A 2004, 101(42):15148-15153

14. Franke $L$, van Bakel $H$, Fokkens $L$, de Jong $E D$, Egmont-Petersen $M$, Wijmenga $C$ : Reconstruction of a functional human gene network, with an application for prioritizing positional candidate genes. Am J Hum Genet 2006, 78(6):1011-1025.

15. Lage K, Karlberg EO, Storling ZM, Olason PI, Pedersen AG, Rigina O, Hinsby AM, Tumer Z, Pociot F, Tommerup N, et al: A human phenomeinteractome network of protein complexes implicated in genetic disorders. Nat Biotechnol 2007, 25(3):309-316.

16. Wu $X$, Jiang $R$, Zhang MQ, Li S: Network-based global inference of human disease genes. Mol Syst Biol 2008, 4:189.

17. Kohler S, Bauer S, Horn D, Robinson PN: Walking the interactome for prioritization of candidate disease genes. Am J Hum Genet 2008, 82(4):949-958

18. Vanunu O, Magger O, Ruppin E, Shlomi T, Sharan R: Associating genes and protein complexes with disease via network propagation. PLOS Comput Biol 2010, 6(1):e1000641.

19. Chen J, Aronow BJ, Jegga AG: Disease candidate gene identification and prioritization using protein interaction networks. BMC Bioinformatics 2009, 10:73.
20. Chen X, Yan GY, Liao XP: A novel candidate disease genes prioritization method based on module partition and rank fusion. OMICS 2010, 14(4):337-356

21. Sun PG, Gao L, Han S: Prediction of human disease-related gene clusters by clustering analysis. Int J Biol Sci 2011, 7(1):61-73.

22. Lin J, Gan CM, Zhang X, Jones S, Sjoblom T, Wood LD, Parsons DW, Papadopoulos N, Kinzler KW, Vogelstein B, et al: A multidimensional analysis of genes mutated in breast and colorectal cancers. Genome Res 2007, 17(9):1304-1318.

23. Carlson MR, Zhang B, Fang Z, Mischel PS, Horvath S, Nelson SF: Gene connectivity, function, and sequence conservation: predictions from modular yeast co-expression networks. BMC Genomics 2006, 7:40.

24. Oldham MC, Horvath S, Geschwind DH: Conservation and evolution of gene coexpression networks in human and chimpanzee brains. Proc Natl Acad Sci U S A 2006, 103(47):17973-17978.

25. Navlakha S, Kingsford C: The power of protein interaction networks for associating genes with diseases. Bioinformatics 2010, 26(8):1057-1063.

26. Salwinski L, Miller CS, Smith AJ, Pettit FK, Bowie JU, Eisenberg D: The database of interacting proteins: 2004 update. Nucleic Acids Res 2004, 32(Database issue):D449-451.

27. Alfarano C, Andrade CE, Anthony K, Bahroos N, Bajec M, Bantoft K, Betel D, Bobechko B, Boutilier K, Burgess $E$, et al: The biomolecular interaction network database and related tools 2005 update. Nucleic Acids Res 2005 33(Database issue):D418-424

28. Aranda B, Achuthan P, Alam-Faruque Y, Armean I, Bridge A, Derow C, Feuermann M, Ghanbarian AT, Kerrien S, Khadake J, et al: The IntAct molecular interaction database in 2010. Nucleic Acids Res 2010, 38(Database issue):D525-531.

29. Pagel $P$, Kovac $S$, Oesterheld M, Brauner B, Dunger-Kaltenbach I, Frishman G, Montrone C, Mark P, Stumpflen V, Mewes HW, et al: The MIPS mammalian protein-protein interaction database. Bioinformatics 2005, 21(6):832-834.

30. Ceol A, Chatr Aryamontri A, Licata L, Peluso D, Briganti L, Perfetto L, Castagnoli L, Cesareni G: MINT, the molecular interaction database: 2009 update. Nucleic Acids Res 2010, 38(Database issue):D532-539.

31. Keshava Prasad TS, Goel R, Kandasamy K, Keerthikumar S, Kumar S, Mathivanan S, Telikicherla D, Raju R, Shafreen B, Venugopal A, et al: Human Protein Reference Database-2009 update. Nucleic Acids Res 2009, 37(Database issue):D767-772

32. Stark C, Breitkreutz BJ, Reguly $T$, Boucher $L$, Breitkreutz A, Tyers M: BioGRID: a general repository for interaction datasets. Nucleic Acids Res 2006, 34(Database issue):D535-539.

33. Matthews L, Gopinath G, Gillespie M, Caudy M, Croft D, de Bono B, Garapati P, Hemish J, Hermjakob H, Jassal B, et al: Reactome knowledgebase of human biological pathways and processes. Nucleic Acids Res 2009, 37(Database issue):D619-622.

34. Cerami EG, Gross BE, Demir E, Rodchenkov I, Babur O, Anwar N, Schultz N, Bader GD, Sander C: Pathway Commons, a web resource for biological pathway data. Nucleic Acids Res 2011, 39(Database issue):D685-690.

35. Jensen $\sqcup$, Kuhn M, Stark M, Chaffron S, Creevey C, Muller J, Doerks T, Julien $\mathrm{P}$, Roth A, Simonovic M, et al: STRING 8-a global view on proteins and their functional interactions in 630 organisms. Nucleic Acids Res 2009, 37(Database issue):D412-416.

36. von Mering $C$, Huynen $M$, Jaeggi $D$, Schmidt S, Bork P, Snel B: STRING: a database of predicted functional associations between proteins. Nucleic Acids Res 2003, 31(1):258-261.

37. Hamosh A, Scott AF, Amberger JS, Bocchini CA, McKusick VA: Online Mendelian Inheritance in Man (OMIM), a knowledgebase of human genes and genetic disorders. Nucleic Acids Res 2005, 33(Database issue): D514-517.

38. Barabasi AL, Oltvai ZN: Network biology: understanding the cell's functional organization. Nat Rev Genet 2004, 5(2):101-113.

39. Chung MY, Lu YC, Cheng NC, Soong BW: A novel autosomal dominant spinocerebellar ataxia (SCA22) linked to chromosome 1p21-q23. Brain 2003, 126(Pt 6):1293-1299.

40. Duenas AM, Goold R, Giunti P: Molecular pathogenesis of spinocerebellar ataxias. Brain 2006, 129(Pt 6):1357-1370

41. Matilla-Duenas A, Sanchez I, Corral-Juan M, Davalos A, Alvarez R, Latorre P: Cellular and molecular pathways triggering neurodegeneration in the spinocerebellar ataxias. Cerebellum 2010, 9(2):148-166. 
42. Schols L, Bauer P, Schmidt T, Schulte T, Riess O: Autosomal dominant cerebellar ataxias: clinical features, genetics, and pathogenesis. Lancet Neurol 2004, 3(5):291-304.

43. Lim J, Hao T, Shaw C, Patel AJ, Szabo G, Rual JF, Fisk CJ, Li N, Smolyar A, Hill DE, et al: A protein-protein interaction network for human inherited ataxias and disorders of Purkinje cell degeneration. Cell 2006, 125(4):801-814.

44. Ikeda Y, Dick KA, Weatherspoon MR, Gincel D, Armbrust KR, Dalton JC, Stevanin G, Durr A, Zuhlke C, Burk K, et al: Spectrin mutations cause spinocerebellar ataxia type 5. Nat Genet 2006, 38(2):184-190.

45. Lorenzo DN, Li MG, Mische SE, Armbrust KR, Ranum LP, Hays TS: Spectrin mutations that cause spinocerebellar ataxia type 5 impair axonal transport and induce neurodegeneration in Drosophila. J Cell Biol 2010, 189(1):143-158.

46. Stankewich MC, Gwynn B, Ardito T, Ji L, Kim J, Robledo RF, Lux SE, Peters LL, Morrow JS: Targeted deletion of betalll spectrin impairs synaptogenesis and generates ataxic and seizure phenotypes. Proc Nat Acad Sci U S A 2010, 107(13):6022-6027.

47. Clarkson YL, Gillespie T, Perkins EM, Lyndon AR, Jackson M: Beta-III spectrin mutation L253P associated with spinocerebellar ataxia type 5 interferes with binding to Arp1 and protein trafficking from the Golgi. Hum Mol Genet 2010, 19(18):3634-3641.

48. Sun G, Tomita H, Shakkottai VG, Gargus JJ: Genomic organization and promoter analysis of human KCNN3 gene. J Hum Genet 2001, 46(8):463-470.

49. Grube S, Gerchen MF, Adamcio B, Pardo LA, Martin S, Malzahn D, Papiol S, Begemann M, Ribbe K, Friedrichs $H$, et al: A CAG repeat polymorphism of KCNN3 predicts SK3 channel function and cognitive performance in schizophrenia. EMBO Mol Med 2011, 3(6):309-319.

50. Jin DK, Hwang HZ, Oh MR, Kim JS, Lee M, Kim S, Lim SW, Seo MY, Kim JH, Kim DK: CAG repeats of CTG18.1 and KCNN3 in Korean patients with bipolar affective disorder. J Affect Disord 2001, 66(1):19-24.

51. Aerts S, Lambrechts D, Maity S, Van Loo P, Coessens B, De Smet F, Tranchevent LC, De Moor B, Marynen P, Hassan B, et al: Gene prioritization through genomic data fusion. Nat Biotechnol 2006, 24(5):537-544.

52. Li Y, Patra JC: Integration of multiple data sources to prioritize candidate genes using discounted rating system. BMC Bioinformatics 2010, 11(Suppl 1):S20.

53. Goh Kl, Cusick ME, Valle D, Childs B, Vidal M, Barabasi AL: The human disease network. Proc Natl Acad Sci U S A 2007, 104(21):8685-8690.

doi:10.1186/1471-2164-12-S3-S25

Cite this article as: Hsu et al:: Prioritizing disease candidate genes by gene interconnectedness-based approach. BMC Genomics 2011 12(Suppl 3):S25.

\section{Submit your next manuscript to BioMed Central and take full advantage of:}

- Convenient online submission

- Thorough peer review

- No space constraints or color figure charges

- Immediate publication on acceptance

- Inclusion in PubMed, CAS, Scopus and Google Scholar

- Research which is freely available for redistribution 